\title{
3 Research Suare \\ CDH1 germline mutations in a Chinese cohort with hereditary diffuse gastric cancer
}

\section{Zhiwen Pan}

Zhejiang Cancer Hospital

\section{Zhixuan Fu}

Zhejiang Cancer Hospital

\section{Cong Luo}

Zhejiang Cancer Hospital

Yejiang Bao

Zhejiang Cancer Hospital

\section{Mingli Wang}

Zhejiang Cancer Hospital

\section{Wenming Cao}

Zhejiang Cancer Hospital

Xiaohong Xu ( $\sim$ zjhzxxh@163.com)

Zhejiang Cancer Hospital https://orcid.org/0000-0003-0134-0579

\section{Research Article}

Keywords: $\mathrm{CDH} 1$, mutation screening, E-cadherin, hereditary diffuse gastric cancer, germline mutation

Posted Date: April 27th, 2021

DOI: https://doi.org/10.21203/rs.3.rs-424936/v1

License: (c) (i) This work is licensed under a Creative Commons Attribution 4.0 International License.

Read Full License 


\section{Abstract \\ Purpose}

Germline mutations in $\mathrm{CDH} 1$ are associated with hereditary diffuse gastric cancer (HDGC) and have been identified in multiple ethnicities. However, $C D H 1$ germline mutations have seldom been documented in Chinese patients with HDGC, and their frequency remains unclear. Here, we aimed to examine the frequency of $\mathrm{CDH} 1$ germline mutations in Chinese patients with HDGC. In total, 285 patients who met the International Gastric Cancer Linkage Consortium 2015 testing criteria of HDGC for $C D H 1$ germline mutations were recruited.

\section{Methods}

All $16 \mathrm{CDH1}$ exons, including neighboring intronic sequences, were amplified using polymerase chain reaction and screened using Sanger sequencing. Variants were analyzed using Mutation Surveyor V4.0, SIFT, and PolyPhen-2 software.

\section{Results}

Three nonsense and nine missense $C D H 1$ germline mutations were identified in 21 of 285 index cases (7.4\%). Two CDH1 germline mutations, N405Y (Asn405Tyr) and W409X (Trp409 Ter) were identified as new variants. In addition, we found that up to $28.6 \%$ of $C D H 1$ mutations in the 21 indicated patients identified as C. $1775 \mathrm{G}>\mathrm{C}$ (E551Q). The frequency of $C D H 1$ mutations was $6.5 \%$ (7/108) in HDGC and $7.9 \%(14 / 177)$ in early onset diffuse gastric cancer (EODGC). The mutation detection rate of $C D H 1$ in males and females was 6.7\% (4/60) and 8.5\% (10/117) in EODGC and 4.6\% (3/65) and 9.3\% (4/43) in HDGC, respectively.

\section{Conclusion}

These data reveal, for the first time, the type and frequency of $\mathrm{CDH1}$ germline mutations in Chinese HDGC, and demonstrate that germline $\mathrm{CDH} 1$ mutations are a noteworthy contributor to the high frequency of HDGC in Chinese.

\section{Introduction}

Gastric cancer (GC) is considered the fifth most common malignancy (Torre et al. 2015) and the third leading cause of cancer-related deaths worldwide (Ferlay et al. 2013). However, an obvious geographical difference exists concerning GC incidence, with the highest incidence rates in East Asian countries (particularly Korea, Mongolia, Japan, and China) and the lowest in Northern America and most parts of Africa (Ferlay et al. 2010). In China, GC was estimated as the second most frequent cancer in men and 
the third most frequent in women; with GC being the second leading cause of cancer-related death in men and women in 2015 (Chen et al. 2016). Accordingly, owing to the large population size and high incidence of GC in the country, nearly $42 \%$ of all GC cases worldwide occur in China alone (Parkin et al. 2005; Chen et al. 2016).

GC is histologically classified into two major types: intestinal and diffuse. The intestinal type is common in the general population and is often related to environmental factors and lifestyle. However, diffuse GC (DGC) is more likely associated with genetic variants (Laurén 1965). Hereditary DGC (HDGC), a subset of DGC, is an autosomal dominant cancer syndrome characterized by signet ring cell carcinomas. HDGC can be caused by heterozygous germline mutations in $C D H 1$, which are regarded as the major genetic cause of HDGC (Guilford et al. 1998). CDH1 maps to chromosome 16q22.1, and encodes the cell adhesion protein E-cadherin, a member of the transmembrane glycoprotein family. E-cadherin is involved in calcium-dependent cell-to-cell adhesion and confers cell polarity. Loss of E-cadherin function is expected to affect the epithelia architecture, cell adhesion, and cell polarity, which are correlated with tumor infiltration and metastasis (Suriano et al. 2003a; van Roy and Berx 2008; Humar and Guilford 2009;). Male patients carrying germline $C D H 1$ mutations have a $70 \%$ risk of developing DGC in their lifetime, whereas $56 \%$ of female patients may develop DGC by the age of 80 (Hansford et al. 2015). Clinical criteria for $C D H 1$ mutation screening were established by the International Gastric Cancer Linkage Consortium (IGCLC) in 1999 (Caldas et al. 1999) and updated in 2010 (Fitzgerald et al. 2010) and 2015 (van der Post et al. 2015a). Germline $C D H 1$ mutations have been detected in approximately $30-50 \%$ of HDGC families fulfilling the original HDGC criteria (Kaurah et al. 2007). To date, more than 100 different germline $C D H 1$ mutations have been reported in various ethnicities worldwide (Hansford et al. 2015). Notably, these germline mutations are dispersed throughout the $\mathrm{CDH} 1 \mathrm{gene}$, and no mutations have been described for HDGC.

Despite the undisputable status of GC as the most common cancer in China, germline $\mathrm{CDH} 1$ mutations are seldom documented in patients with HDGC. Here, we present the first report on the type and spectrum of $\mathrm{CDH1}$ germline mutations in the largest index cases of Chinese patients with HDGC to date.

\section{Methods}

\section{Patients}

In total, 285 index cases meeting the IGCLC 2015 clinical criteria for HDGC were recruited between February 2013 and July 2018. All diagnoses were confirmed from pathology reports at Zhejiang Cancer Hospital. Clinical information (e.g., age and sex) was obtained from clinical records. This study was conducted in accordance with the recommendations of the Ethics Committee of the Zhejiang Cancer Hospital. The protocol was approved by the Ethics Committee of Zhejiang Cancer Hospital (approval no. IRB-2019-172). All subjects provided written informed consent in accordance with the Declaration of Helsinki. Patients who did not meet the 2015 clinical criteria for HDGC were excluded from the study.

\section{CDH1 genetic testing}


Genomic DNA was extracted from peripheral blood leukocytes using the QIAamp DNA Mini Kit (Qiagen, Valencia, CA, USA), according to the manufacturer's instructions. The entire coding sequence and flanking intronic portions of the $C D H 1$ gene were amplified by polymerase chain reaction (PCR) and sequenced via Sanger sequencing using standard procedures. The primers were designed by the authors and are presented in Table 1. PCR was carried out in a $20 \mu \mathrm{L}$ volume (detailed conditions available upon request). The amplicons were sequenced on an ABI 3730XL sequencer (Life Technologies, Carlsbad, CA, USA).

Table 1

Primers used for polymerase chain reaction amplification

\begin{tabular}{|c|c|c|c|}
\hline Exon & Forward 5邓-3区 & Reverse 5囚-3】 & Product size \\
\hline E1E2 & GCAAAGCACCTGTGAGCTT & GCGTAAATTCCAAGGGGTGTC & 1242 \\
\hline E3 & AGCACAAGGAAGTCATCCT & TGTCATAACTGGTGGAAGTGC & 616 \\
\hline E4E5 & AGCAAAGGGTCTCATTGGT & GCTCCTCATGTGTTCAGAGC & 737 \\
\hline E6 & AGCCTAGGAAGGTGTGGCA & CCAAGAAGTTCTGTCCGTAGG & 380 \\
\hline E7E8 & TATCCCTCAGGGCAGAATTGG & GCCATCTCAAGATGCTTGC & 822 \\
\hline E9 & GGAGGATTGCTTGAGCCCA & ACTGCAAATCCCACATGGTCC & 522 \\
\hline E10 & GCAGATTTGAGAAGCCATGGT & AGAGGCAGCACATCAGACC & 637 \\
\hline E11 & GACCTCAGGTGATCTGCCCA & ATTTGGGTGACGGATACCCT & 561 \\
\hline E12 & TGTAAAACGGCCAGAGACCT & ACCTTTGGAGCAAGGCCTC & 585 \\
\hline E13 & AAAACCCAAGCAGCTCTGC & GCTGGCATAACTTGGGAGTC & 496 \\
\hline E14 & ACCGACTTCAGGGATGTGAG & TGAGCTTCTCTGTGCCTCAGC & 554 \\
\hline E15 & CTCACAATCCTTTGGGCCA & GACACAACTCCTCCTGAGCTT & 412 \\
\hline E16 & GTTCACTGCTCCGTGGTGTG & AAACCACCAGCAACGTGAT & 408 \\
\hline
\end{tabular}

\section{Mutation analysis and in silico prediction}

Variants were analyzed using the Mutation Surveyor V4.0 software package (Software Genetics, State College, PA, USA). Missense mutations were analyzed using SIFT (Kumar et al. 2009) and PolyPhen-2 (Adzhubei et al. 2010) software to predict the potential functional effects of amino acid substitutions.

\section{Results}

\section{Categorization of HDGC cases}

The IGCLC clinical criteria for testing germline mutations of $C D H 1$ in HDGC, as revised in 2015, are as follows: (i) two GC cases regardless of age, with at least one confirmed as DGC; (ii) one DGC case before the age of 40 years without a family history of GC; and (iii) a personal or family history of DGC and 
lobular breast cancer, with one diagnosis occurring before the age of 50 years. According to these criteria for genetic screening, all cases in the present study were classified into two categories: (a) 108 index cases with a family history of HDGC, and (b) 177 patients with early onset diffuse gastric cancer (EODGC). Testing for $\mathrm{CDH1}$ germline mutations was performed in 285 unrelated Chinese patients.

\section{Detection of germline $\mathrm{CDH} 1$ mutations}

Among the 285 patients subjected to $C D H 1$ mutation analysis, 21 index cases were detected as $C D H 1$ germline mutation carriers (7.4\%) (Table 2). Among the 21 mutation carriers, 14 were classified as EODGC and seven had a family history of HDGC. Twelve non-synonymous mutations were identified in these patients (Table 3), including three truncating mutations (nonsense mutations) and nine missense mutations. Notably, two of the 12 mutations identified were novel: c. $1225 \mathrm{G}>\mathrm{A}$ (Trp409 Ter) and c.1213A $>T$ (Asn405Tyr). Three of the other germline mutations have been reported previously in HDGC cases: c.489C > A (Cys163X), c.1018A > G (Thr340Ala), and c.1118C > T (Pro373Leu). Five additional synonymous mutations were identified in $\mathrm{CDH} 1$ exons, which were previously registered in the National Center for Biotechnology Information (NCBI) dbSNP database (https://www.ncbi.nlm.nih.gov/snp/) (Table 4).

Table 2

Summary of $\mathrm{CDH} 1$ germline mutation types identified in the study population

\begin{tabular}{|c|c|c|c|c|c|}
\hline \multirow{2}{*}{$\begin{array}{l}2015 \\
\text { criteria }\end{array}$} & \multirow[t]{2}{*}{ Definition } & \multirow{2}{*}{$\begin{array}{l}\text { Index } \\
\text { Cases }\end{array}$} & \multicolumn{2}{|l|}{ Mutations } & \multirow{2}{*}{$\begin{array}{l}\text { Total } \\
\text { no. of } \\
\text { patients } \\
\text { (\%) }\end{array}$} \\
\hline & & & Missense & Stop & \\
\hline 1 & $\begin{array}{l}\text { Two or more GC cases, regardless of age, with at } \\
\text { least one with confirmed DGC }\end{array}$ & 108 & 5 & 1 & $\begin{array}{l}7 \\
(6.5 \%)\end{array}$ \\
\hline 2 & DGC onset at $<40$ years & 177 & 7 & 2 & $\begin{array}{l}14 \\
(7.9 \%)\end{array}$ \\
\hline Total & & 285 & 9 & 3 & $\begin{array}{l}21 \\
(7.4 \%)\end{array}$ \\
\hline
\end{tabular}


Table 3

Index cases with $\mathrm{CDH} 1$ germline mutations

\begin{tabular}{|c|c|c|c|}
\hline ID & Region & Mutation & Mutation type/pathogenicity \\
\hline 20 & E8 & c. $1018 \mathrm{~A}>\mathrm{G} /$ p. $340 \mathrm{~T}>\mathrm{A}$ & possible pathogenic \\
\hline 100 & E8 & c. $1018 \mathrm{~A}>\mathrm{G} /$ p. $340 \mathrm{~T}>\mathrm{A}$ & possible pathogenic \\
\hline 127 & E4 & c. 489 C $>$ A/p. 163 C $>X$ & pathogenic \\
\hline 140 & E16 & c. $2638 \mathrm{G}>\mathrm{A} / \mathrm{p} .880 \mathrm{E}>\mathrm{K}$ & possible pathogenic \\
\hline 148 & E12 & c. $1888 \mathrm{C}>\mathrm{G} /$ p. $630 \mathrm{~L}>\mathrm{V}$ & possible pathogenic \\
\hline 154 & E8 & c. $1118 \mathrm{C}>\mathrm{T} / \mathrm{p} .373 \mathrm{P}>\mathrm{L}$ & possible pathogenic \\
\hline 171 & E2 & c. $76 \mathrm{G}>\mathrm{A} / \mathrm{p} .26 \mathrm{E}>\mathrm{K}$ & benign \\
\hline 244 & E9 & c. $1225 \mathrm{G}>\mathrm{A} /$ p. $409 \mathrm{~W}>\mathrm{X} \dagger$ & pathogenic \\
\hline 260 & E8 & c. $1018 A>$ G/p.340T >A & possible pathogenic \\
\hline 276 & E11 & c. $1651 \mathrm{G}>$ C/p.551E $>Q$ & possible pathogenic \\
\hline 302 & E9 & c. $1296 \mathrm{C}>\mathrm{G} / \mathrm{p} .432 \mathrm{~N}>\mathrm{K}$ & possible pathogenic \\
\hline 323 & E11 & c. $1651 \mathrm{G}>$ C/p.551E $>Q$ & possible pathogenic \\
\hline 340 & E11 & c. $1651 \mathrm{G}>$ C/p.551E $>$ Q & possible pathogenic \\
\hline \multirow[t]{2}{*}{347} & E3 & c. $187 \mathrm{C}>\mathrm{T} / \mathrm{p} .63 \mathrm{R}>\mathrm{X}$ & pathogenic \\
\hline & E12 & c. $1888 \mathrm{C}>\mathrm{G} /$ p. $630 \mathrm{~L}>\mathrm{V}$ & possible pathogenic \\
\hline 350 & E11 & c. $1651 \mathrm{G}>$ C $/$ p. $551 \mathrm{E}>\mathrm{Q}$ & possible pathogenic \\
\hline 371 & E9 & c. $1213 \mathrm{~A}>\mathrm{T} / \mathrm{p} .405 \mathrm{~N}>\mathrm{Y} \dagger$ & possible pathogenic \\
\hline 405 & E8 & c. $1018 \mathrm{~A}>\mathrm{G} /$ p. $340 \mathrm{~T}>\mathrm{A}$ & possible pathogenic \\
\hline 410 & E12 & c. $1888 \mathrm{C}>\mathrm{G} /$ p. $630 \mathrm{~L}>\mathrm{V}$ & possible pathogenic \\
\hline 413 & E8 & c. $1019 \mathrm{C}>\mathrm{T} / \mathrm{p} .340 \mathrm{~T}>\mathrm{M}$ & possible pathogenic \\
\hline 449 & E11 & c. $1651 \mathrm{G}>$ C/p.551E $>Q$ & possible pathogenic \\
\hline 465 & E11 & c. $1651 \mathrm{G}>$ C/p.551E $>$ Q & possible pathogenic \\
\hline
\end{tabular}


Table 4

CDH1 synonymous variants in exons of HDGC in the study population

\begin{tabular}{|c|c|c|c|c|}
\hline Site & $C D H 1$ variant & Mutation type & Proportion of patients & dbSNP \\
\hline E4 & c.393 C > T(S131S) & silent & $1 / 167$ & rs145430811 \\
\hline E13 & c. $2076 \mathrm{~T}>\mathrm{C}(\mathrm{A} 692 \mathrm{~A})$ & silent & $137 / 167$ & rs1801552 \\
\hline E13 & c. 2079 C > T(G693G) & silent & $1 / 167$ & rs771993728 \\
\hline E13 & c. 2103 C > T(V701V) & silent & $1 / 167$ & rs730881656 \\
\hline E14 & c. $2253 \mathrm{C}>\mathrm{T}(\mathrm{N} 751 \mathrm{~N})$ & silent & $33 / 167$ & rs33964119 \\
\hline
\end{tabular}

We also performed in silico analyses to assess the effect of missense mutations on E-cadherin function. Web-based SIFT (Kumar et al. 2009) and Polyphen-2 (Adzhubei et al. 2010) software were used to predict whether the amino acid change conferred by variants might alter protein structure and function. The analysis indicated that the mutation E26K is less likely to be a pathogenic variant. However, other mutations were considered pathogenic based on in silico analyses.

\section{Discussion}

To our knowledge, our series of 285 index cases of HDGC with germline $C D H 1$ mutations is the largest reported from China to date. The penetrance of $C D H 1$ germline mutations in DGC is reported to be nearly 100\% (Barber et al. 2008; Rogers et al. 2008). Therefore, it is important to identify $C D H 1$ mutation carriers that benefit from gene mutation screening and the implementation of cancer risk reduction strategies. The findings of the present study provide valuable clinical information that is expected to assist clinical and laboratory-based genetic cancer experts in effectively managing patients with germline mutations.

To date, few $C D H 1$ mutations have been reported in HDGCs from Chinese patients (Jiang et al. 2004; Zhang et al. 2006; More et al. 2007). In the current study, we described 12 CDH1 germline mutations in HDGCs. The pathogenic $C D H 1$ germline variant $\mathrm{C} 163 \mathrm{X}$ was identified in a patient diagnosed with signet ring cell carcinoma of the stomach at 38 years, whose mother also suffered from GC. This mutation generated a premature stop codon at position 163 of the E-cadherin (C163X) protein and was thus considered pathogenic. The $\mathrm{C} 163 \mathrm{X}$ mutation has been previously described in two separate studies (Kluijt et al. 2012; van der Post et al. 2015b) and was detected for the first time in Chinese patients with HDGC in the present study. The R63X mutation was identified in a 40 year old female patient. She also carried an L630V CDH1 mutation. The germline mutation L630V was also detected in two index male patients with EODGC. The germline mutation W409X in the $C D H 1$ gene was reported in a 42-year-old female patient, which was first identified in a patient with HDGC and was a novel $C D H 1$ germline mutation.

The T340A variant was first identified by Kim et al. (2000) and has been reported in more than four distinct patients or families (Oliveira et al. 2002; Suriano et al. 2003b; van der Post et al. 2015b), including a Chinese patient (Zhang et al. 2006). In our study, we described the variant in four unrelated female 
patients. The nucleotide variant c.1019C > T caused a T340M amino acid variation, which was identified in a female index. Functional analysis has suggested a pathogenic role for the T340A mutation in the Ecadherin protein (Suriano et al. 2006; van Roy and Berx 2008). In contrast, the results of van der Post et al. (van der Post et al. 2015b) did not present the variant as a pathogenic mutation; however, the study lacked consistent GC histories and segregation analyses. Thus, based on our findings and previous reports, we classified this variant as a pathogenic mutation.

Roviello et al. (2007) first reported the missense mutation P373L in an Italian family. We detected this mutation in a Chinese patient with HDGC and classified it as pathogenic based on in silico analysis. The N405Y was identified in 37 year old male patient, which was first reported in HDGC cases and as a novel mutation.

Notably, the E551Q mutation was detected in six unrelated patients, composed of approximately $2.1 \%$ of the cohort (6/285) and $28.6 \%$ of all mutation carriers identified in this study $(6 / 21)$. As the germline mutations identified to date were distributed across the $C D H 1$ gene and no hotspot mutation has been reported, this finding was rather unexpected. Therefore, the findings suggest that E551Q may be a hotspot mutation in Chinese patients with HDGC.

The detection rate of $\mathrm{CDH} 1$ germline mutations in Chinese patients with HDGC is considered low, as only a few have been reported in several distinct Chinese families with HDGC (Jiang et al. 2004; Zhang et al. 2006; More et al. 2007); although, no large cohorts have been examined systematically. The purported rarity of the $C D H 1$ mutation in Chinese patients with HDGC may be related to an insufficient number of patients evaluated or a lack of studies on this disease, and is not associated with the actual prevalence of HDGC in China. The overall $C D H 1$ mutation rate in countries with a high risk of GC, such as Italy (22.2\%) (Corso et al. 2011), Japan (15.4\%) (Yamada et al. 2011), and Korea (Kim et al. 2013; Choi et al. 2014) is lower than that in low-risk countries such as Canada, the USA, and the UK (51.6\%); however, $\mathrm{CDH} 1$ mutations in high-risk GC countries are generally composed of dominant missense mutations (Corso et al. 2012). In our study, the detection rate for the $C D H 1$ mutation was $7.4 \%$ (21/285), which was lower than that in low-risk GC countries. In addition, the major type of mutation identified in our study was missense, constituting $75 \%$ (9/12) of the mutations and $25 \%(3 / 12)$ of non-missense mutations. Apart from missense mutations, no deletions/duplications have been identified in Chinese patients with HDGC. There are two possible reasons for this: one is that the deletion/duplication mutations are infrequent in Chinese HDGC patients; second, the sample size may not have been sufficient to detect deletions/duplications. These findings concur with the conclusions of a meta-analysis by Corso et al. (2012).

CDH1 germline mutations in patients with EODGC without a family history have been documented in literature (Choi et al. 2014); however, the actual frequency in this subset of DGC has not been described in the Chinese population. The detection rate of the $C D H 1$ mutation in European patients with EODGC was 4.9-10\%, which was lower than that reported in HDGC with a family history of GC in each study (Oliveira et al. 2002; Benusiglio et al. 2013; Benusiglio et al. 2015; Hansford et al. 2015). Among areas with a high 
incidence of $\mathrm{GC}$, the detection rate of $\mathrm{CDH} 1$ germline mutations in EODGC was $8 \%$ (South Korea) (Kim et al. 2013) and 9.5\% (Italy) (Corso et al. 2011). In the present study, among 177 unrelated cases, 14 tested positive for $C D H 1$ mutations, with a $7.9 \%$ (14/177) detection rate of $C D H 1$ germline mutations. The $C D H 1$ germline mutation rate in Chinese patients with EODGC was similar to that in patients from Korea and Italy.

It has been reported that patients with EOGC and those with GC at older ages clearly display different clinical and pathological features. For example, patients under the age of 40 are more often female, whereas older patients are mostly male (Brenner et al. 2000; Windham et al. 2002; Lim et al. 2003). Among the 177 patients with EODGC in the present study, female patients outnumbered male patients $177 / 60$, similar to the proportions among patients with EOGC. The detection rate of the $C D H 1$ mutation in EODGC was similar in female patients, and that in male patients was $8.5 \%(10 / 117)$ and $6.7 \%(4 / 60)$. Although there were more female than male patients with EOGC, a similar $C D H 1$ mutation rate was noted between them. This indicates that the $\mathrm{CDH} 1$ mutation may not be the main cause of early onset gastric cancer in women than in men. Maeta et al. (1995) speculated that the development of GC in young women may be markedly influenced by natural, biological, and hormonal factors. However, this hypothesis requires further investigation.

It has been estimated that male $C D H 1$ mutation carriers have a $67-70 \%$ risk of developing DGC, whereas females exhibit a $56-83 \%$ risk by 80 years old (Hansford et al. 2015). Therefore, it is important to identify mutation carriers before they develop lethal symptomatic DGC, which has a survival rate below $20 \%$ (Lynch et al. 2005). Based on these findings, despite the low frequency of $C D H 1$ germline mutations in China, we recommend gene screening for people at risk and highlight the importance of early identification of $C D H 1$ mutation carriers, especially for individuals with EODGC, to provide suitable intervention before the emergence of lethal symptoms.

Our study has a limitation in that we did not screen for the $C D H 1$ gene for large-fragment deletions with multiplex ligation-dependent probe amplification. We plan to conduct this study in future studies. To our knowledge, this study is the first to describe the frequency of $\mathrm{CDH1}$ germline mutations in Chinese patients with HDGC. The detection rate of $\mathrm{CDH} 1$ germline mutations in Chinese patients with HDGC was $7.4 \%$. These results confirmed that patients with HDGC from high-risk areas, such as China, show a lower incidence of $C D H 1$ germline mutations compared to individuals from low-risk regions. These data should provide useful assistance for genetic counseling and management of patients at risk (Corso et al. 2014).

\section{Declarations}

Acknowledgments: We would like to thank Editage (www.editage.com) for English language editing.

Funding: This study was supported by the Medical and Health Science Project of Zhejiang Province (2015KYA033, 2016139538, 2017KY021). The funders had no role in the study design, data collection and analysis, decision to publish, or preparation of the manuscript. 
Conflict of Interest: The authors declare that they have no conflict of interest.

Data Availability Statement: All datasets analyzed for this study are included in the manuscript/supplementary files.

Code availability: Not applicable

Author Contributions: Conceptualization: Zhiwen Pan, Cong Luo, and Xiaohong Xu; Data curation: Mingli Wang, Wenming Cao, Zhiwen Pan and Zhixuan Fu; Writing - original draft: Zhiwen Pan and Xiaohong Xu. Supervision and writing - review \& editing: Zhiwen Pan, Zhixuan Fu, Cong Luo, Yejiang Bao, Mingli Wang, Wenming Cao, Xiaohong Xu.

Ethics approval: The protocol of the study was approved by the Ethics Committee of the Zhejiang Cancer Hospital (approval no. IRB-2017-47). All subjects provided written informed consent in accordance with the Declaration of Helsinki.

Consent to participate: Informed consent was obtained from all individual participants included in the study.

Consent to publish: All individuals participating in the study have provided the consent to publish the data

\section{References}

Adzhubei IA, Schmidt S, Peshkin L, Ramensky VE, Gerasimova A, Bork P, et al (2010) A method and server for predicting damaging missense mutations. Nat Methods 7:248-249.

https://doi.org/10.1038/nmeth0410-248

Barber ME, Save V, Carneiro F, Dwerryhouse S, Lao-Sirieix P, Hardwick RH, et al (2008) Histopathological and molecular analysis of gastrectomy specimens from hereditary diffuse gastric cancer patients has implications for endoscopic surveillance of individuals at risk. J Pathol 216(3):286-294.

https://doi.org/10.1002/path.2415

Benusiglio PR, Malka D, Rouleau E, Pauw AD, Buecher B, Noguès $C$, et al (2013) CDH1 germline mutations and the hereditary diffuse gastric and lobular breast cancer syndrome: a multicentre study. J Med Genet 50(7):486-489. https://doi.org/10.1136/jmedgenet-2012-101472

Benusiglio PR, Colas C, Rouleau E, Uhrhammer N, Romero P, Remenieras A, et al (2015) Hereditary diffuse gastric cancer syndrome: improved performances of the 2015 testing criteria for the identification of probands with a CDH1 germline mutation. J Med Genet 52(8):563-565.

https://doi.org/10.1136/jmedgenet-2015-103153

Brenner H, Bode G, Boeing H (2000) Helicobacter pylori infection among offspring of patients with stomach cancer. Gastroenterology 118(1):31-35. https://doi.org/10.1016/S0016-5085(00)70411-2 
Caldas C, Carneiro HT, Lynch F, Lynch J, Yokota H, Yokota GL, et al (1999) Familial gastric cancer: overview and guidelines for management. J Med Genet 36(12):873-880.

Chen W, Zheng R, Baade P, Zhang S, Zeng H, Bray F, et al. (2016) Cancer statistics in China, 2015. CA Cancer J Clin 66(2):115-132. https://doi.org/10.3322/caac.21338

Choi H, Ki C, Suh S, Kim J (2014) Presymptomatic identification of CDH1 germline mutation in a healthy Korean individual with family history of gastric cancer. Ann Lab Med 34(5):386-389.

https://doi.org/0.3343/alm.2014.34.5.386

Corso G, Pedrazzani C, Pinheiro H, Fernandes E, Marrelli D, Rinnovati A, et al (2011) E-cadherin genetic screening and clinico-pathologic characteristics of early onset gastric cancer. Eur J Cancer 47(4):631639. https://doi.org/10.1016/j.ejca.2010.10.011

Corso G, Marrelli D, Pascale V, Vindigni C, Roviello F (2012) Frequency of CDH1 germline mutations in gastric carcinoma coming from high- and low-risk areas: metanalysis and systematic review of the literature. BMC Cancer 6:12:8. https://doi.org/10.1186/1471-2407-12-8

Corso G, Figueiredo J, Biffi R, Trentin C, Bonanni B, Feroce I, et al (2014) E-cadherin germline mutation carriers: clinical management and genetic implications. Cancer Metastasis Rev 33(4):1081-1094. https://doi.org/10.1007/s10555-014-9528-y

Ferlay F, Parkin D, Steliarova-Foucher E (2010) Estimates of cancer incidence and mortality in Europe in 2008. Eur J Cancer 46(4):765-781. https://doi.org/10.1016/j.ejca.2009.12.014

Ferlay F, Ervik M, Dikshit R, Eser S, Mathers C. GLOBOCAN 2012 v1.0, Cancer incidence and mortality worldwide: IARC CancerBase No.11, International Agency for Research on Cancer, 2013.

Fitzgerald RC, Hardwick R, Huntsman D, Carneiro F, Caldas C (2010) Hereditary diffuse gastric cancer: updated consensus guidelines for clinical management and directions for future research. $J$ Med Genet 47(7):436-444. https://doi.org/10.1136/jmg.2009.074237

Guilford P, Hopkins J, Harraway J, Mcleod M, Mcleod N, Harawira P, et al (1998) E-cadherin germline mutations in familial gastric cancer. Nature 392(6674):402-405. https://doi.org/10.1038/32918

Hansford S, Kaurah P, Li-Chang H, Woo M, Senz J, Pinheiro H, et al (2015) Hereditary diffuse gastric cancer syndrome: $\mathrm{CDH} 1$ mutations and beyond. JAMA Oncol 1(1):23-32.

https://doi.org/10.1001/jamaoncol.2014.168

Humar B, Guilford P (2009) Hereditary diffuse gastric cancer: a manifestation of lost cell polarity. Cancer Sci 100(7):1151-1157. https://doi.org/10.1111/j.1349-7006.2009.01163.x

Jiang Y, Wan YL, Wang ZJ, Zhao B, Zhu J, Huang YT (2004) Germline E-cadherin gene mutation screening in familial gastric cancer kindreds. Chinese J Surg 42(15):914-917. 
Kaurah P, MacMillan A, Boyd N, et al (2007) Founder and recurrent CDH1 mutations in families with hereditary diffuse gastric cancer. JAMA 297(21):2360-2372. https://doi.org/10.1001/jama.297.21.2360

Kim HC, Wheeler JMD, Kim JC, llyas M, Beck NE, Kim BS, et al (2000) The E-cadherin gene (CDH1) variants T340A and L599V in gastric and colorectal cancer patients in Korea. Gut 47(2):262-267. https://doi.org/10.1136/gut.47.2.262

Kim S, Chung JW, Jeong TD, Park YS, Lee JH, Ahn JY, et al (2013) Searching for E-cadherin gene mutations in early onset diffuse gastric cancer and hereditary diffuse gastric cancer in Korean patients. Fam Cancer 12(3):503. https://doi.org/10.1007/s10689-012-9595-6

Kluijt I, Siemerink E, Ausems M, van Os T, de Jong D, Simões-Correia J, et al (2012) CDH1-related hereditary diffuse gastric cancer syndrome: clinical variations and implications for counseling. Int $\mathrm{J}$ Cancer 131(2):367-376. https://doi.org/10.1002/ijc.26398

Kumar P, Henikoff S, Ng P (2009) Predicting the effects of coding non-synonymous variants on protein function using the SIFT algorithm. Nat Protoc 4:1073-1081. https://doi.org/10.1038/nprot.2009.86

Laurén P (1965) The two histological main types of gastric carcinoma: diffuse and so-called intestinal type carcinoma. An attempt at a histo-clinical classification. Acta Pathol Microbiol Scandinavica 64:3149. https://doi.org/10.1111/apm.1965.64.1.31

Lim S, Lee HS, Kim HS, Kim YI, Kim WH (2003) Alteration of E-cadherin-mediated adhesion protein is common, but microsatellite instability is uncommon in young age gastric cancers. Histopathology 42(2):128-136. https://doi.org/10.1046/j.1365-2559.2003.01546.x

Lynch HT, Grady W, Suriano G, Huntsman D (2005) Gastric cancer: new genetic developments. J Surg Oncol 90(3):114-133. https://doi.org/10.1002/jso.20214

Maeta M, Yamashiro H, Oka A, Tsujitani S, Ikeguchi M, Kaibara N (1995) Gastric cancer in the young, with special reference to 14 pregnancy-associated cases: analysis based on 2,325 consecutive cases of gastric cancer. J Surg Oncol 58(3):191-195. https://doi.org/10.1002/jso.2930580310

More H, Humar B, Weber W, Ward R, Christian A, Lintott C, et al (2007) Identification of seven novel germline mutations in the human E-cadherin (CDH1) gene. Hum Mutat 28(2):203.

https://doi.org/10.1002/humu.9473

Oliveira C, Bordin M, Grehan N, Huntsman D, Suriano G, Machado J, et al (2002) Screening E-cadherin in gastric cancer families reveals germline mutations only in hereditary diffuse gastric cancer kindred. Hum Mutat 19(5):510-517. https://doi.org/10.1002/humu.10068

Parkin DM, Bray F, Ferlay J, Pisani P. Global cancer statistics, 2002 (2005) Ca Cancer J Clin 55(2):74-108. https://doi.org/10.3322/canjclin.55.2.74 
Rogers WM, Dobo E, Norton J Van Dam J, Jeffrey RB, Huntsman et al (2008) Risk-reducing total gastrectomy for germline mutations in E-cadherin $(\mathrm{CDH} 1)$ : pathologic findings with clinical implications. Am J Surg Pathol 32(6):799-809. https://doi.org/10.1097/PAS.0b013e31815e7f1a

Roviello F, Corso G, Pedrazzani C, Marrelli D, De Falco G, Berardi A, et al (2007) Hereditary diffuse gastric cancer and E-cadherin: description of the first germline mutation in an Italian family. Eur $\mathrm{J}$ Surg Oncol 33(4):448-451. https://doi.org/10.1016/j.ejso.2006.10.028

Suriano G, Oliveira C, Ferreira P, Machado JC, Bordin MC, De Wever O, et al (2003a) Identification of CDH1 germline missense mutations associated with functional inactivation of the E-cadherin protein in young gastric cancer probands. Hum Mol Gen 12(5):575-582. https://doi.org/10.1093/hmg/ddg048

Suriano G, Oliveira MJ, Huntsman D, Mateus AR, Ferreira P, Casares F, et al (2003b) E-cadherin germline missense mutations and cell phenotype: evidence for the independence of cell invasion on the motile capabilities of the cells. Hum Mol Genet 12(22):3007-3016. https://doi.org/10.1093/hmg/ddg316

Suriano G, Seixas S, Rocha J, Seruca R (2006) A model to infer the pathogenic significance of CDH1 germline missense variants. J Mol Med 84(12):1023-1031. https://doi.org/10.1007/s00109-006-0091-z

Torre LA, Bray F, Siegel RL, Ferlay J, Lortet-Tieulent J, Jemal, A (2015) Global cancer statistics, 2012. Ca A Cancer J Clin 65(2):69-90. https://doi.org/10.3322/caac.21262

van der Post RS, Vogelaar IP, Carneiro F, Guilford P, Huntsman D, Hoogerbrugge N, et al (2015a) Hereditary diffuse gastric cancer: updated clinical guidelines with an emphasis on germline $\mathrm{CDH} 1$ mutation carriers. J Med Genet 52(6):361-374. https://doi.org/10.1136/jmedgenet-2015-103094

van der Post RS, Vogelaar IP, Manders P, van der Kolk LE, Cats A, van Hest LP, et al (2015b) Accuracy of hereditary diffuse gastric cancer testing criteria and outcomes in patients with a germline mutation in CDH1. Gastroenterology 149(4):897-906. https://doi.org/10.1053/j.gastro.2015.06.003

van Roy F, Berx G (2008) The cell-cell adhesion molecule E-cadherin. Cell Mol Life Sci 65(23):3756-3788. https://doi.org/10.1007/s00018-008-8281-1

Windham TC, Termuhlen PM, Ajani JA, Mansfield PF (2002) Adenocarcinoma of the stomach in patients age 35 years and younger: no impact of early diagnosis on survival outcome. J Surg Oncol 81(3):118124. https://doi.org/10.1002/jso.10157

Yamada H, Shinmura K, Ito H, Kasami M, Sasaki N, Shima H, et al (2011) Germline alterations in the $\mathrm{CDH} 1$ gene in familial gastric cancer in the Japanese population. Cancer Sci 102(10):1782-1788. https://doi.org/10.1111/j.1349-7006.2011.02038.x

Zhang Y, Liu X, Fan Y, Ding J, Xu A, Zhou X, et al (2006) Germline mutations and polymorphic variants in MMR, E-cadherin and MYH genes associated with familial gastric cancer in Jiangsu of China. Int $J$ Cancer 119(11):2592-2596. https://doi.org/10.1002/ijc.22206 